\title{
NEUTRINOLESS DOUBLE BETA DECAY AND NEW PHYSICS IN THE NEUTRINO SECTOR
}

\author{
H.V. KLAPDOR-KLEINGROTHAUS, H. PÄS \\ Max-Planck-Institut für Kernphysik, P.O. Box 103980, D-69029 Heidelberg \\ E-mail: klapdor@gustav.mpi-hd.mpg.de \\ E-mail: Heinrich.Paes@mpi-hd.mpg.de
}

\begin{abstract}
Neutrinoless double beta decay belongs to the most sensitive tools for the search of new physics beyond the standard model. The recent half life limit of the Heidelberg-Moscow experiment implies restrictive bounds on the absolute mass scale in the neutrino sector. Possible improvements by the GENIUS project provide a unique possibility to reconstruct the neutrino mass spectrum. Further constraints on new interactions in the neutrino sector are given in a model-independent way. Consequences for neutrino anomalies and theories beyond the standard model such as left-right symmetric models, R-parity violating SUSY and leptoquarks are discussed. The potential of double beta decay experiments in the search for WIMP dark matter is reviewed.
\end{abstract}

\section{Introduction}

Double beta decay 1.2 corresponds to two single beta decays occuring in one nucleus and converts a nucleus $(\mathrm{Z}, \mathrm{A})$ into a nucleus $(\mathrm{Z}+2, \mathrm{~A})$. While even the standard model (SM) allowed process emitting two antineutrinos

$$
{ }_{Z}^{A} X \rightarrow{ }_{Z+2}^{A} X+2 e^{-}+2 \bar{\nu}_{e}
$$

is one of the rarest processes in nature with half lives in the region of $10^{21-24}$ years, more interesting is the search for the neutrinoless mode $(0 \nu \beta \beta)$,

$$
{ }_{Z}^{A} X \rightarrow{ }_{Z+2}^{A} X+2 e^{-}
$$

which viplates lepton number by two units and thus implies physics beyond the $\mathrm{SM}$.

The most sensitive experiment so far, the Heidelberg-Moscow experiment Q⿴囗十 1 is searching for the $0 \nu \beta \beta$ decay of ${ }^{76} \mathrm{Ge}$. The results after $31 \mathrm{~kg}$ y measuring time using digital pulse shape analysis correspond to a conservative half life limit of 4

$$
\begin{array}{ll}
T_{1 / 2}^{0 \nu \beta \beta}>1.8 \cdot 10^{25} y & (90 \% \text { C.L. }), \\
T_{1 / 2}^{0 \nu \beta \beta}>3.0 \cdot 10^{25} y & (68 \% \text { C.L. }) .
\end{array}
$$

To render possible a further breakthrough in search for neutrino masses and physics beyond the SM, GENIUS, an experiment operating a large amount 


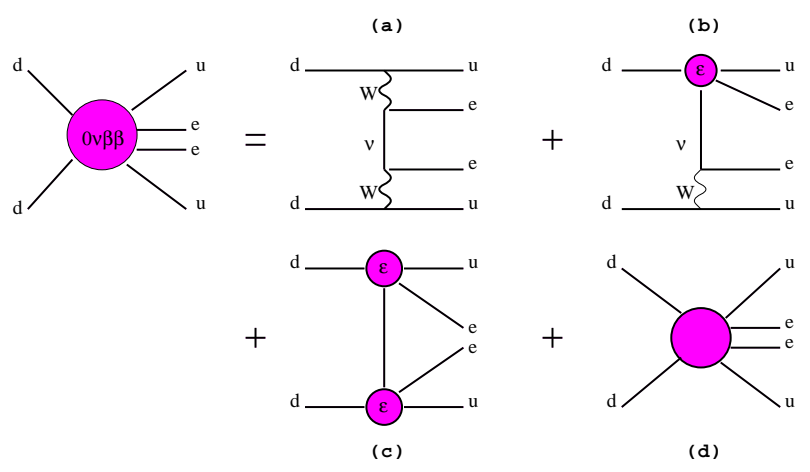

Figure 1. Feynman graphs of the general double beta rate: The contribution a) corresponds to the neutrino mass mechanism with SM interactions and is discussed in the context of neutrino oscillations in section 2. The contributions b) - d) include new neutrino interactions and are discussed in section 3).

of naked Ge-detectors in a liquid nitrogen shielding, has been proposed 1 目. Operating 288 enriched ${ }^{76} \mathrm{Ge}$ detectors with a total mass of 1 ton inside a nitrogen tank of $\sim 12 \mathrm{~m}$ height and diameter, one could access half lifes of $T_{1 / 2}^{0 \nu \beta \beta}=6 \cdot 10^{27} y$ after one year of measurement. A ten ton version would reach a final sensitivity of $T_{1 / 2}^{0 \nu \beta \beta}=6 \cdot 10^{29} \mathrm{y}$ within 10 years of measurement time.

\section{Neutrino masses and oscillations}

The search for $0 \nu \beta \beta$ decay exchanging a massive left-handed Majorana neutrino between two SM vertices (contribution a) in fig. 1) at present provides the most sensitive approach to determine an absolute neutrino mass and also a unique possibility to distinguish between the Dirac or Majorana nature of the neutrino. With the recent half life limit of the Heidelberg-Moscow experiment the following conservative limit on the effective neutrino mass

$$
\begin{aligned}
& \left\langle m_{\nu}\right\rangle=\sum_{i} U_{e i}^{2} m_{i} \leq 0.36 e V \quad(90 \% \text { C.L. }) \\
& \left\langle m_{\nu}\right\rangle=\sum_{i} U_{e i}^{2} m_{i} \leq 0.28 \mathrm{eV} \quad(68 \% \text { C.L. })
\end{aligned}
$$

can be deduced. Here the sum extends over light mass eigenstates $m_{i}$ only. The GENIUS project could access effective neutrino masses down to $10^{-2} \mathrm{eV}$ or even $10^{-3} \mathrm{eV}$ in the 1 ton or 10 ton version, respectively. Since in specific 
models of neutrino masses the quantity $\langle m\rangle$ can be related to the oscillation parameters $\Delta m^{2}$ and $\sin ^{2} 2 \theta$, these bounds imply restrictive bounds on the neutrino mass spectrum, which in most cases are more stringent than the bounds from precision measurements of the CMB by MAP and Planck. The extreme cases discussed here are degenerate and hierarchical models (for a detailed discussion see $\mathrm{U}$ ).

Degenerate models: Such models have been proposed to get a large mass scale for neutrinos acting as hot dark matter and at the same time accomodate two of the three neutrino anomalies (solar, atmospheric \& LSND neutrinos). In this framework neutrino masses up to (few) eV are predicted. Thus large mixing (vacuum or MSW LMA oscillations) and strong cancellations are required to be consistent with eq. 1. E.g., maximal cancellation, the MSW LMA bestfit and a $\Lambda$ CHDM model with a Hubble constant of $h \simeq 0.5$ implies a value of $\langle m\rangle=0.15 \mathrm{eV}$ just below the present limit.

Hierarchical models: Such models give less optimistic predictions for $0 \nu \beta \beta$ decay. However, assuming the MSW LMA solution, still sizable contributions up to $\langle m\rangle=($ few $) 10^{-2}$ in the reach of GENIUS are possible. Since $0 \nu \beta \beta$ decay is most sensitive in the large $\Delta m^{2}$ region of the LMA solution, it may provide complementary informations to the search for day-night effects in solar neutrinos.

For a super-heavy left-handed neutrino a bound of $\left\langle m_{H}\right\rangle=\left(\sum_{j} \frac{U_{e j}^{2}}{m_{j}}\right)^{-1}>$ $9 \cdot 10^{7} \mathrm{GeV}$ can be deduced, with heavy mass eigenstates $m_{j}$. This constraint makes a heavy neutfino unobservable at linear colliders except in the most contrived scenarios $\mathrm{B}$.

\section{New interactions}

Besides the exchange of massive Majorana neutrinos between two SM vertices, a variety of theories beyond the SM predicts new lepton number violating interactions contributing to neutrinoless double beta decay, leading to the idea to construct the general double beta decay rate allowed by Lorentzinvariance 0.11 . This approach allows to constrain lepton number violating parameters in arbitrary models.

For the long range part of the decay rate with two separable vertices and light neutrino exchange in between (contributions b) and c) in fig. 1), one has to consider the Lorentz-invariant contractions of six projections with defined helicity both for the leptonic $\left(j_{\alpha}\right)$ and hadronic $\left(J_{\alpha}\right)$ current. The general Lagrangian can be written in terms of effective couplings $\epsilon_{\beta}^{\alpha}$, which correspond to the pointlike vertices at the Fermi scale so that Fierz rearrangement is

cosmo99: submitted to World Scientific on October 24, 2018 
applicable:

$$
\mathcal{L}=\frac{G_{F}}{\sqrt{2}}\left\{j_{V-A}^{\mu} J_{V-A, \mu}^{\dagger}+\sum_{\alpha, \beta}^{\prime} \epsilon_{\alpha}^{\beta} j_{\beta} J_{\alpha}^{\dagger}\right\}
$$

with the combinations of hadronic and leptonic Lorentz currents of defined helicity $\alpha, \beta=V-A, V+A, S-P, S+P, T_{L}, T_{R}$. The prime indicates that the sum runs over all contractions allowed by Lorentz-invariance, except for $\alpha=\beta=V-A$. Here $\epsilon_{\alpha}^{\beta}$ denotes the strength of the non-SM couplings. For the helicity suppressed terms proportional to the (from below) unconstrained neutrino mass no limit can be derived and terms proportional $\left(\epsilon_{\alpha}^{\beta}\right)^{2}$ can be neglected. The limits on the remaining non-SM couplings derived in s-wave approximation and evaluated "on axis" are 9 (here and in the following $90 \%$ C.L. $): \epsilon_{V+A}^{V+A}<6 \cdot 10^{-7}, \epsilon_{V-A}^{V+A}<4 \cdot 10^{-9}, \epsilon_{S+P}^{S+P}<9 \cdot 10^{-9}, \epsilon_{S-P}^{S+P}<9 \cdot 10^{-9}$, $\epsilon_{T_{R}}^{T_{R}}<1 \cdot 10^{-9}, \epsilon_{T_{L}}^{T_{R}}<6 \cdot 10^{-10}$. These bounds e.g. exclude the possibility to fake the LSND anomaly (and this way accomodate for all neutrino anomalies with only three neutrinos) via the lepton number violating reaction $\nu_{e} u_{L} \rightarrow d_{R} e^{+}$in a model-independent way 10 .

For the short range part the hadronic currents have to be contracted with leptonic currents $j_{\alpha}=\bar{e} \mathcal{O}_{\alpha} e^{C}$, where $\mathcal{O}_{\alpha}$ denotes the operators of defined helicty discussed above. In this case the general Lagrangian is

$$
\begin{array}{r}
\mathcal{L}=\frac{G_{F}^{2}}{2} m_{P}^{-1}\left\{\epsilon_{1} J J j+\epsilon_{2} J^{\mu \nu} J_{\mu \nu} j+\epsilon_{3} J^{\mu} J_{\mu} j+\epsilon_{4} J^{\mu} J_{\mu \nu} j^{\nu}+\epsilon_{5} J^{\mu} J j_{\mu}\right. \\
\left.+\epsilon_{6} J^{\mu} J^{\nu} j_{\mu \nu}+\epsilon_{7} J J^{\mu \nu} j_{\mu \nu}+\epsilon_{8} J_{\mu \kappa} J^{\nu \kappa} j_{\nu}^{\mu}\right\},
\end{array}
$$

where indices $\alpha$ have been suppressed. Since no fundamental tensors exist in renormalizable theories and since the leptonic tensor current vanishes in the s-wave approximation, the contributions proportional to $\epsilon_{4} \epsilon_{6}, \epsilon_{7}, \epsilon_{8}$ can be neglected. The remaining terms are constrained as follows 11 : $\epsilon_{1}<3 \cdot 10^{-7}$, $\epsilon_{2}<2 \cdot 10^{-9}, \epsilon_{3}<4 \cdot 10^{-8} / 1 \cdot 10^{-8}(V \mp A V \mp A / V \mp A V \pm A), \epsilon_{5}<2 \cdot 10^{-7}$.

\section{Left-right symmetry, R-parity violation, Leptoquarks}

In this section we apply the general discussion above to specific theories of physics beyond the SM.

Left-Right-Symmetric Models: In left-right symmetric models the lefthandedness of weak interactions is explained as due to the effect of different symmetry breaking scales in the left- and in the right-handed sector. $0 \nu \beta \beta$ decay proceeds through exchange of the heavy right-handed partner of the

cosmo99: submitted to World Scientific on October 24, 2018 
ordinary neutrino between right-handed $\mathrm{W}$ vertices, leading to a limit of

$$
m_{W_{R}} \geq 1.4\left(\frac{m_{N}}{1 T e V}\right)^{-(1 / 4)} \mathrm{TeV} .
$$

Including a theoretical limit obtained from considerations of vacuum stability 12 one can deduce an absolute lower limit on the right-handed $\mathrm{W}$ mass of 3

$$
m_{W_{R}} \geq 1.4 \mathrm{TeV} \text {. }
$$

Supersymmetry: While in the minimal supersymmetric extension (MSSM) $\mathrm{R}$-parity is assumed to be conserved, there are no theoretical reasons for $R_{p}$ conservation and several GUT and Superstring models require R-parity violation in the low energy regime. In this case $0 \nu \beta \beta$ decay can occur through Feynman oraphs involving the exchange of superpartners as well as $\not_{P}$-couplings $\lambda^{\prime}$ 14,16.17, 18, 29. The half-life limit of the Heidelberg-Moscow experiment leads to bounds in a multidimensional parameter space 4.17

$$
\lambda_{111}^{\prime} \leq 4 \times 10^{-4}\left(\frac{m_{\tilde{q}}}{100 G e V}\right)^{2}\left(\frac{m_{\tilde{g}}}{100 G e V}\right)^{1 / 2}
$$

(for $m_{\tilde{d}_{R}}=m_{\tilde{u}_{L}}$ ), which are the sharpest limits on $R_{P}$ SUSY.

$0 \nu \beta \beta$ decay is not only sensitive to $\lambda_{111}^{\prime}$. Taking into account the fact that the SUSY partners of the left- and right-handed quark states can mix with each other, new diagrams appear in which the neutrino-mediated double beta decay is accompanied by SUSY exchange in the vertices 15.18. A calculation of previously neglected tensor contributions to the decay rate allows to derive improved limits on different combinations of $\lambda^{\prime} 19$. Assuming the supersymmetric mass parameters of order $100 \mathrm{GeV}$, the half life limit of the HeidelbergMoscow Experiment implies: $\lambda_{113}^{\prime} \lambda_{131}^{\prime} \leq 3 \cdot 10^{-8}, \lambda_{112}^{\prime} \lambda_{121}^{\prime} \leq 1 \cdot 10^{-6}$

In addition, stringent bounds on coupling products can be derived directly from the effective mass bound eq. 1, since R-parity violating interactions will produce neutrino Majorana masses on loop level. It implies $21 \lambda_{133}^{\prime} \lambda_{133}^{\prime}<$ $5 \cdot 10^{-8}, \lambda_{132}^{\prime} \lambda_{123}^{\prime}<1 \cdot 10^{-6}, \lambda_{122}^{\prime} \lambda_{122}^{\prime}<3 \cdot 10^{-5}, \lambda_{133} \lambda_{133}<9 \cdot 10^{-7}, \lambda_{132} \lambda_{123}<$ $2 \cdot 10^{-5}, \lambda_{122} \lambda_{122}<2 \cdot 10^{-4}$.

In the case of R-parity conserving SUSY, based on a theorem proven in 20 , the $0 \nu \beta \beta$ mass limits can be converted in sneutrino Majorana mass term limits being more restrictive than what could be obtained in inverse neutrinoless double heta decay and single sneutrino production at future linear colliders (NLC) 20.

Leptoquarks: Leptoquarks are scalar or vector particles coupling both to leptons and quarks, which appear naturally in GUT, extended Technicolor or Compositeness models. The mixing of different multiplets by introducing a leptoquark-Higgs coupling would lead to a contribution to $0 \nu \beta \beta$ decay

cosmo99: submitted to World Scientific on October 24, 2018 
22. Combined with the half-life limit of the Heidelberg-Moscow experiment bounds on effective couplings can be derived 23. Assuming only one lepton number violating $\Delta L=2$ LQ-Higgs coupling unequal to zero and the leptoquark masses not too different, one can derive from this limit either a bound on the LQ-Higgs coupling

$$
Y_{L Q-H i g g s}=(\mathrm{few}) \cdot 10^{-6}
$$

or a limit excluding leptoquarks with masses in the range of $\mathcal{O}(200 \mathrm{GeV})$. Assuming $Y_{L Q} \sim \mathcal{O}(1)$ leptoquark masses should be larger than (few) 10 $\mathrm{TeV}$.

\section{Violations of the equivalence principle and Lorentz invariance}

Special relativity and the equivalence principle can be considered as the most basic foundations of the theory of gravity. However, string theories may allow for or even predict the violation of these laws. Such effects in the neutrino sector have been extensively studied in the framework of neutrino oscillations 24. A typical feature of the violation of Lorentz invariance (VLI) is that different species of matter may have characteristic maximal attainable velocities. The quantity $\delta v$ provides an observable for VLI. The corresponding quantity describing violations of the equivalence principle (VEP) is the difference of characteristic couplings $\delta g$ to the gravitational potential $\phi$. While previous studies of neutrino oscillations are restricted to the region of large mixing of velocity/gravitational and flavor eigenstates, $0 \nu \beta \beta$ decay provides a bound in the previously unconstrained region of zero mixing 25: $\delta v<4 \cdot 10^{-16}$, $\phi \delta g<4 \cdot 10^{-16}$.

\section{WIMP Dark Matter Search with Double Beta Experiments}

Weakly interacting masssive particles (WIMPs) such as the lightest supersymmetric particle (LSP) are major candidates for the cold component of nonbaryonic dark matter in the universe. Due to its low background properties double beta technology can also find applications in the search for direct detection of WIMPs. The Heidelberg-Moscow Experiment, without being specially designed for this purpose, gave the most stringent limits on WIMPs for several years 26. New results with $0.69 \mathrm{~kg}$ y of measurement reached a background level of $0.042 \mathrm{cts} /(\mathrm{kg} \mathrm{d} \mathrm{keV})$ in the region between $15 \mathrm{keV}$ and $40 \mathrm{keV}$. The derived limit excludes WIMPS with masses greater than $13 \mathrm{GeV}$ and cross sections as low as $1.12 \cdot 10^{-5} \mathrm{pb}$. These are the most stringent limits

cosmo99: submitted to World Scientific on October 24, 2018 
on spin-independent interactions using only raw data 27. The GENIUS experiment would allow to test almost the entire MSSM parameter space already in a first step using only $100 \mathrm{~kg}$ of enriched or even natural Ge 1.5 .

\section{Summary}

Neutrinoless double beta decay and dark matter search belong to the most sensitive approaches with great perspectives to test particle physics beyond the SM.

The possibilities to use $0 \nu \beta \beta$ decay (and the most sensitive HeidelbergMoscow experiment) for constraining neutrino masses, new interactions beyond the standard model, and violations of Lorentz invariance and the equivalence principle have been reviewed. Experimental limits on $0 \nu \beta \beta$ decay are not only complementary to accelerator experiments, neutrino oscillations and cosmological precision measurements, but at least in some cases competitive or superior to the best existing or planned approaches. Direct WIMP detection experiments can compete with recent and future accelerator experiments in the search for SUSY and experiments using double beta technology belong to the most promising approaches in this field of research. A further large breakthrough, both for double beta decay and dark matter search, will be possible realizing the GENIUS proposal, which would improve the obtained limits by up to 1-2 orders of magnitude.

\section{References}

1. H.V. Klapdor-Kleingrothaus, Int. J. Mod. Phys. A 13 (1998) 3953; Proc. Lepton and Baryon Number Violation, IOP Bristol \& Philadelphia 1999, Eds. H.V. Klapdor-Kleingrothaus, I. Krivosheina, 251-301

2. W.C. Haxton, G.J. Stephenson, Progr. Part. Nucl. Phys. 12 (1984) 409; M. Moe, P. Vogel, Annual Review of Nucl. Part. Science 44 (1994) 247; M. Doi, T. Kotani, E. Takasugi, Progr. Theor. Phys. Suppl. 83 (1985) 1

3. H.V. Klapdor-Kleingrothaus, H. Päs (Eds.): Beyond the Desert-Accelerator and Non-Accelerator Approaches, Proc. Int. Workshop on Particle Physics beyond the Standard Model, Castle Ringberg, June 8-14, 1997, IOP Publ., Bristol, Philadelphia

4. HEIDELBERG-MOSCOW collab., Phys. Rev. Lett. 83 (1999) 41; priv. comm.

5. H.V. Klapdor-Kleingrothaus, in 3 ; J. Hellmig, H.V. KlapdorKleingrothaus, Z. Phys. A 359 (1997) 351; H.V. Klapdor-Kleingrothaus, M. Hirsch, Z. Phys. A 359 (1997) 361; H.V. Klapdor-Kleingrothaus,

cosmo99: submitted to World Scientific on October 24, 2018 
J. Hellmig, M. Hirsch, J. Phys. G 24 (1998) 483; H.V. KlapdorKleingrothaus, Y. Ramachers, Eur. Phys. J. A 3 (1998) 85

6. H.V. Klapdor-Kleingrothaus, L. Baudis, G. Heusser, B. Majorovits, H. Päs, hep-ph/9910205

7. H.V. Klapdor-Kleingrothaus, H. Päs, A.Y. Smirnov, to be published

8. G. Belanger, Proc. Lepton and Baryon Number Violation, see $\square$

9. H. Päs, M. Hirsch, S.G. Kovalenko, H.V. Klapdor-Kleingrothaus, Phys. Lett. B 453 (1999) 194

10. S. Bergmann, H.V. Klapdor-Kleingrothaus, H. Päs, to be published

11. H. Päs, M. Hirsch, H.V. Klapdor-Kleingrothaus, S.G. Kovalenko, to be published

12. R.N. Mohapatra, Phys. Rev D 34 (1986) 3457

13. M. Hirsch, H.V. Klapdor-Kleingrothaus, O. Panella, Phys. Lett. B 374 (1996) 7

14. M. Hirsch, H.V. Klapdor-Kleingrothaus, S.G. Kovalenko, Phys. Rev. Lett. 75 (1995) 17

15. K. S. Babu, R. N. Mohapatra, Phys.Rev.Lett. 75 (1995) 2276

16. M. Hirsch, H.V. Klapdor-Kleingrothaus, S. Kovalenko, Phys. Lett. B $352(1995) 1$

17. M. Hirsch, H.V. Klapdor-Kleingrothaus, S. Kovalenko, Phys. Rev. D 53 (1996) 1329

18. M. Hirsch, H.V. Klapdor-Kleingrothaus, S.G. Kovalenko, Phys. Lett. B $372(1996) 181$

19. H. Päs, M. Hirsch, H.V. Klapdor-Kleingrothaus, Phys. Lett. B 459 (1999) 450

20. M. Hirsch, H.V. Klapdor-Kleingrothaus, S.G. Kovalenko, Phys. Lett. B 398 (1997) 311 and 403 (1997) 291; M. Hirsch, H.V. KlapdorKleingrothaus, S.G. Kovalenko, Phys.Rev. D 57 (1998) 1947;

21. G. Bhattacharyya, H.V. Klapdor-Kleingrothaus, H. Päs, Phys.Lett. B463 (1999) $77-82$

22. M. Hirsch, H.V. Klapdor-Kleingrothaus, S.G. Kovalenko, Phys. Lett. B 378 (1996) 17

23. M. Hirsch, H.V. Klapdor-Kleingrothaus, S.G. Kovalenko Phys. Rev. D 54 (1996) R4207

24. C. Leung, hep-ph/0002073 and references therein

25. H.V. Klapdor-Kleingrothaus, H. Päs, U. Sarkar, Eur. Phys. J A5 (1999) 3

26. HEIDELBERG-MOSCOW collab., Phys. Lett. B 336 (1994) 141

27. HEIDELBERG-MOSCOW collab., Phys. Rev. D 59 (1998) 022001 